\title{
Towards enhancing the detection of adulteration in bioactive food products
}

\author{
Charles Odilichukwu R. Okpala
}

Independent academic research practice consultant, c/o Aguiyi Ironsi Street, Off Finbars Road, Umuahia, Abia State, Nigeria.

E-mail: charlesokpala@gmail.com

DOI: $10.31665 /$ JFB.2019.6183

Received: June 15, 2019; Revised received \& accepted: June 27, 2019

Citation: Okpala, C.O.R. (2019). Towards enhancing the detection of adulteration in bioactive food products. J. Food Bioact. 6: 6-9.

\begin{abstract}
This contribution aims to add a voice towards enhancing the detection of adulteration in bioactive food products. Bioactive foods-strongly associated with plant-based chemical compounds, have been shown to boost human immune function and promote health/wellbeing. On the other hand, adulteration generally makes food products to fall short of legal standards, become impure, unsafe and not wholesome. Given that food products can get adulterated either accidentally, intentionally, metallically or naturally, foods that contain bioactive compounds will not be exempted. Adulteration and adulterants therefore pose serious danger to both authenticity and quality of bioactive foods. Considering this challenge, rapid detection methods are needed to enhance the authenticity of bioactive product quality as well as consumer confidence/safety.
\end{abstract}

Keywords: Adulteration; Adulterant; Bioactive foods; Detection methods; Microscopic analysis.

US Federal Food, Drug and Cosmetic Act declares a food is adulterated 'if any valuable constituent had been in whole or in part omitted...or if any substance has been substituted wholly or in part...or if damage or inferiority has been concealed...or if any substance has been reduced its quality or strength or make it appear of greater value than it is' (Everstine et al., 2013). Thus, food adulteration either adds/removes any substance to or from food to affect natural composition and quality (Munikrishnan, 2013). The mixture/substitution of substances largely considered as inferior and/or removal of ingredients to intentionally debase food quality altogether can render a product toxic and thus, affect human health to deprive essential nutrient required for proper development and growth. Indeed, consumers would feel cheated when food gets adulterated, as they have insufficient knowledge about purity and quality of food particles (Jha, 2016). Notably, food adulteration presents itself in different forms, that is, accidental, intentional, metallic and or natural forms. Accidental (unintentional) form can occur due to either ignorance or lack of facility to sustain food quality, for example, inappropriate food handling. Intentional (non-accidental) form can occur due to the pursuit for financial gain that can be found in various practices, for example, addition of water to an already liquid-type food product (Munikrishnan, 2013). Metallic form can occur due to the accidental/intentional addition of metallic substance. Metallic, in this instance, broadly refers to such chemical element(s) as iron $(\mathrm{Fe})$, lead $(\mathrm{Pb})$, mercury $(\mathrm{Hg})$ and Tin $(\mathrm{Sn})$. Typical examples of metallic form of adulteration include lead from water, mercury from effluents, tin from cans, and so on (Adhikari, 2018). Natural form can occur due to the presence of certain organic compound(s)/radical(s) capable of posing (serious/severe) health hazard(s)/risk(s) and neither accidentally nor intentionally added, for example, toxic varieties of pulses (Munikrishnan, 2013).

For emphasis, the deliberate/intentional addition, adulteration, misrepresentation, substitution and/or tampering of food and food ingredients or provision of false/misleading statements about a given food product for economically motivated reason(s), amounts to food fraud (Tähkäpää et al., 2015). Particularly, adulterant can refer to any substance present within other substances, which compromises efficacy, effectiveness and/or safety of the said substance. In addition, methods of food adulteration can also involve mixing, substitution, concealing quality, decomposed food, misbranding, false labeling as well as toxicants (Adhikari, 2018; Everstine et al., 2013; Munikrishnan, 2013). Further, food product can be considered to become adulterated based on the following: (a) not meeting nature of quality/substance for consumer demand; (b) to possess either cheaper or inferior substance; (c) to prepare/keep/pack un- 
Table 1. Some typical examples of foods/food items together with its respective bioactive chemicals/compounds

\begin{tabular}{lll}
\hline Foods/Food Items & Bioactive chemicals/compounds & References \\
\hline Vegetables, e.g. Broccoli & Tocopherols, Carotenoids & Rao, 2003 \\
Millet grain & Phenolics & Shahidi and Peng, 2018 \\
Soy bread & Isoflavones & Shahidi and Peng, 2018 \\
Grapes & Flavonoids, Phenolics & Shahidi and Peng, 2018 \\
Raspberry, Blueberry & Anthocyanins, Phenolics & Shahidi and Peng, 2018 \\
Orange juice & Flavonoids & Shahidi and Peng, 2018 \\
Fish oil & Omega-3 fatty acid & Rao 2003 \\
Palm oil & Tocopherols, Carotenoids & Rao, 2003 \\
Tumeric & Eugenol, Capscicin & Rao, 2003 \\
Black beans & Phenolics, Flavonoids & Shahidi and Peng, 2018 \\
Various baby foods & Carotenoids & Shahidi and Peng, 2018 \\
\hline
\end{tabular}

der unclean/unworthy conditions that result in contamination; (d) to contain substances that either depreciates and or injuriously affects human health; (e) part or whole substitution of original nature of product by abstracting vital portion(s) of substance; and (f) imitation of some other food product/substance. Further, there are a number of causes that lead to food adulteration, like: (a) profit motive of traders (as part of business strategy); (b) food insecurity (to quantitatively increase production/sales); (c) increased urbanization (to maximize profit by fewer investment from food items); (d) high population demands (increased food demand by population and its changing trends); (e) illiteracy level of general public (low consciousness); (f) lack of effective food laws; and (g) lack of government initiatives (Adhikari, 2018). Besides, some countries have promulgated laws to help prevent adulteration of foods. Broadly, such laws have been understood to encompass authorization given to responsible personnel, degree/nature of penalties, food analyses/ standards as well as sampling procedures (Jha, 2016).

On the other hand, the beneficial components in functional foods have been associated with such (specific) terms like phytochemicals, functional components, as well as bioactive components (Pennington, 2002). Actually, bioactive foods were initially defined as "foods, food ingredients or dietary supplements that demonstrate specific health or medical benefits, which includes prevention and treatment of disease beyond basic nutritional functions". Primarily, bioactive foods are fortified with such nutrients as vitamins, minerals and nutraceuticals (Sharma and Singh, 2010). Further, bioactive foods can possess such phytochemicals with biological functions, and largely classified either as nonstarch polysaccharides (cellulose, hemicellulose, etc.), antioxidants (polyphenolic compounds, flavonoids, carotenoids, etc.), detoxifying agents (reductive acids, phenolds, flavones, carotenoids, etc.) and others (alkaloids, volatile flavor compounds, amines, etc.) (Rao, 2003). Specifically, bioactive compounds (also called bioactives) not only show effects on human health but also distinguish from nutrients and non-nutrients, with bioavailability that partially overlap with nutritional and pharmacological principle. Anyways, bioavailability (simply called bioactivity) encompasses range of bioaccessibility, metabolic and physiological activity (Shahidi and Peng, 2018). Comprising of wide variation of chemical function(s) and structure(s) however, bioactive foods-strongly associated with plant-based chemicals, possess the capacity to boost immune function as well as promote effective health/wellbeing. Some typical examples of foods/food items together with its respective bioactive chemicals/compounds are shown in Table 1. All these food items largely plant-based, from grapes, raspberry, vegetables, orange juice to various baby foods, contain bioactive chemicals/ compounds (Rao, 2003; Shahidi and Peng, 2018) that play active/ key role in health promotion and disease reduction (Shahidi, 2009; Watson and Preedy, 2010). More so, berry fruits particularly rich source of (natural) antioxidants, are represented by vitamin $\mathrm{C}$ and such polyphenols like anthocyanins, phenolic acids, flavonols and tannins. As demonstrated by clinical research, the bioavailability of those naturally occurring compounds can significantly exceed the health gains/merits exhibited by their corresponding supplements already commercially obtainable in pharmaceutical form(s) (Szajdek and Borowska, 2008). Designed to meet four consumer demands of convenience, price, sample proposition and taste however, bioactive foods perform on the principle that both cancer and heart disease are concerns of fatigue and stress, hence the need for them to be part of daily diet to prevent coronary artery disease (CAD) (Sharma and Singh, 2010).

Whilst the intentional tampering of food is not a recent drawback, there is reported evidence where food adulteration/fraud brought about severe casualties in many parts of the globe, for example, China, Italy, Spain, Ukraine, among others (Tähkäpää et al., 2015). Largely, adulteration targets both food products that possess high commercial value and those produced in high tonnage (Cordella et al., 2002). Therefore, any food that contains bioactive compounds will not be exempted (from both adulterants and adulteration). For example, adulteration in food products of bioactive importance include: addition of or dilution with water/ sugar (fruit juice), addition of corn/cane sugar (honey), addition of cheaper seed oil (vegetable oil), as well as addition of cheaper berries (berry jam). Also, soy can be adulterated by genetic modification (in the European Union) (Cordella et al., 2002). Besides, some serious (global) issues have arisen from the adulteration of bioactive foods. For example, adulteration of olive oil with hazelnut oil caused EU countries economic loss of approx. $€ 4$ million/ year. Asian honey was banned in Europe because illegal antibiotics were found present. Phthalate plasticizer di-2-ethylhexyl phthalate as clouding agent, attracted attention of (various) regulatory authorities/general public when it was used to replace replace palm oil in foods (Lohumi et al., 2015). Even between 1820s and 1850s, the use of water was reported as an adulterant to increase the bulk/ weight in olive oil. Also, ground rice, mustard seed husks and sawdust were used to increase the overall bulk/weight of cayenne 


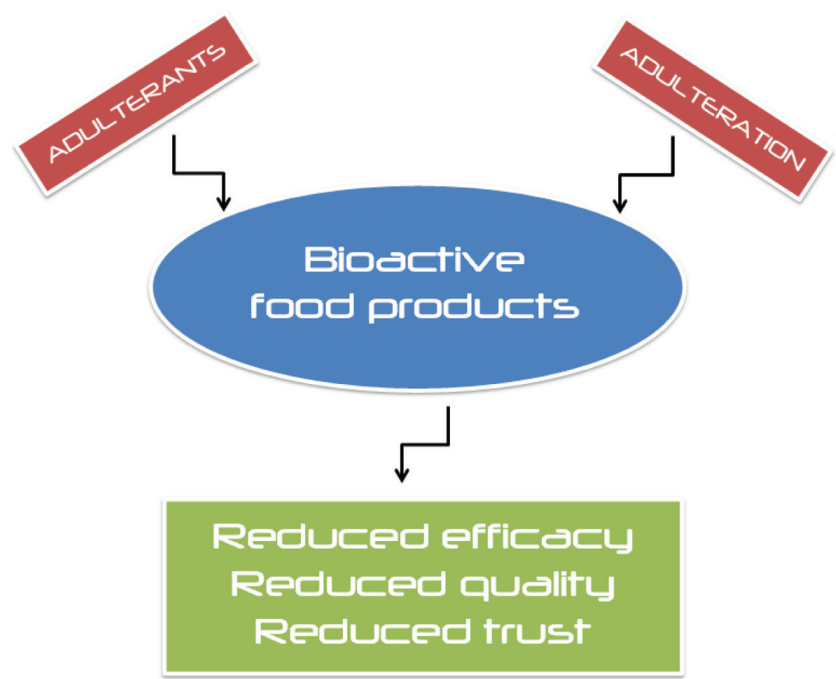

Figure 1. Adulterants and adulteration can pose serious danger in reducing the efficacy, quality and trust associated with bioactive food products.

pepper. Also, previously used dried (tea) leaves were also used to increase the overall bulk of (high quality) tea (Ellis et al., 2012). Detection of adulterants and adulteration in foods therefore has already been long in existence. Another instance is the relabeling of country-of-origin - a serious (trade-related) concern with regards to honey products (Everstine et al., 2013). Clearly, both adulterants and adulteration can pose serious danger to reduce the efficacy, quality and trust associated with bioactive food products (Figure 1). Thus, bioactive food products seriously require its needed attention given the role it plays in boosting human immune function and promoting health/wellbeing (Shahidi, 2009).

Considering the abovementioned challenges (and others not mentioned here) that confront adulterants and adulteration in food products of bioactive importance, more rapid detection methods is therefore warranted. In fact, there is a greater/urgent need in increasing pace/rapidity in detecting adulterants and adulteration in food products of bioactive importance. This will allow for strengthened consumer confidence in food product quality, assure consumer safety, increase product authenticity, and make more robust the quality assurance/control systems. A start could be a displeased former employee to blow the whistle to uncover product adulteration, for example, within a given bioactive food company. From literature however, it is believed that to detect adulteration could technically pose challenges/difficulties because sometimes, the adulterant may hold approximately the same/similar chemical composition of food product in which it is found/included (Cordella et al., 2002) especially in foods with bioactive compounds. Further, Cordella et al. (2002) considers three way-outs to detect adulteration, which include: (a) determining ratio between some chemical constituents with the assumption that these ratios are constant component of the particular food; (b) searching for specific marker within the product that is either chemical constituent or morphological component that proves the food product is adulterated; (c) global approach of food products that employs analytical techniques derived from physical analysis that considers the entire/whole samples to show effects of adulteration based on changes in physiochemical properties. Besides, there is need for analytical methods that would deliver widespread access, not so expensive and yet affordable. In addition, Cordella et al. (2002) in- dicates that these analytical methods operate at microscopic levels with specialist facilities. They include: high-performance liquid chromatography (HPLC), gas chromatography (GC), gas chromatography - mass spectrometry (GCMS), fourier transform infrared spectrometry (FT-IR), gas chromatography/fourier transform infrared spectrometry (GC/FTIR), atomic absorption spectroscopy/ atomic emission spectrometry (AAS/AES), inductively coupled plasma atomic emission spectroscopy (ICP-AES), inductively coupled plasma mass spectrometry (ICP-MS), isotope ratio mass spectrometry (IRMS), gas chromatography-isotope ratio mass spectrometry (GC-IRMS), gas chromatography combustion isotope ratio mass spectrometry (GC-C-IRMS), differential scanning calorimetry (DSC), infrared radiation (IR), Mid IR (mid-infrared spectroscopy), and nuclear magnetic resonance (NMR). Whilst HPLC can detect that cheaper berries have been added to berry jam, $\mathrm{GC} /{ }^{13} \mathrm{C}$ IRMS can detect that sugar has been added to fruit juice. Whilst ${ }^{13} \mathrm{C}$ IRMS can detect that cane/corn sugar has been added to honey, GCMS can also detect that cheaper seed oil was added to vegetable oil (Cordella et al., 2002). FT-IR spectra can discriminate two juice types, for example, between pure grapefruit and orange juice types based on differences/stretches emerging within respective spectral features and flavonoid components (Ellis et al., 2012). To wrap up, the effective use of these (abovementioned) analytical/microscopic specialist facilities certainly should be facilitated/promoted, so as to intensify laboratory activities as well as strengthen the rapid detection of adulteration/adulterants in bioactive food products across (many parts of) the globe.

\section{Acknowledgments}

Author has received no funding support for this current work. Thanks to Dott. Giacomo Sardo of IRBIM-CNR Mazara del Vallo - Italy, for his help with the figure.

\section{Conflict of interest}

Author hereby declares there is no competing interest associated with this current work.

\section{References}

Adhikari, S. (2018). Food Adulteration, Types of Food Adulteration and Mitigation Measures. Public Health Notes, Reported August 5, 2018. (https://www.publichealthnotes.com/food-adulteration-types-of-foodadulteration-and-mitigation-measures/;Accessed 01June 2019,09.29 h GMT).

Cordella, C., Moussa, I., Martel, A.-C., Sbirrazzuoli, N., and Lizzani-Cuvelier, L. (2002). Recent Developments in Food Characterization and Adulteration Detection: Technique-Oriented Perspectives. J. Agric. Food Chem. 50: 1751-1764.

Ellis, D.I., Brewster, V.L., Dunn, W.B., Allwood, J.W., Golovanov, A.P., and Goodacre, R. (2012). Fingerprinting food: current technologies for the detection of food adulteration and contamination. ChemSocRev 41: 5706-5727.

Everstine, K., Spink, J., and Kennedy, S. (2013). Economically Motivated Adulteration (EMA) of Food: Common Characteristics of EMA Incidents. J. Food Protect. 76(4): 723-735.

Jha, S.N. (2016). Rapid Detection of Food Adulterants and Contaminants - Theory and Practice. Academic Press Elsevier, London, United Kingdom, p. 278. ISBN: 978-0-12-420084-5.

Lohumi, S., Lee, S., Lee, H., and Cho, B.-K. (2015). A review of vibrational spectroscopic techniques for the detection of food authenticity and 
adulteration. Trends Food Sci. Technol. 46(1): 85-98.

Munikrishnan, V. (2013). Effects of food adulteration on human health FnBnews.com, Reported on 02 January 2013. 08.00 AM (IST) (http:// www.fnbnews.com/Top-News/Effects-of-food-adulteration-on-human-health; Accessed 02 June 2019, 23.13 h GMT).

Pennington, J.A.T. (2002). Food Composition Databases for Bioactive Food Components. J. Food Composition Analysis 15: 419-434.

Rao, B.N. (2003). Bioactive phytochemicals in Indian foods and their potential in health promotion and disease prevention. Asia Pacific J. Clin Nutr. 12(1): 9-22.

Shahidi, F. (2009). Nutraceuticals and functional foods: Whole versus processed foods. Trends Food Sci. Technol. 20(9): 376-387.

Shahidi, F., and Peng, H. (2018). Bioaccessibility and bioavailability of phe- nolic compounds. J. Food Bioactives 4: 11-68.

Sharma, R., and Singh, R.B. (2010). Bioactive Foods and Nutraceutical Supplementation Criteria in Cardiovascular Protection. The Open Nutraceuticals J. 3: 141-153.

Szajdek, A., and Borowska, E.J. (2008). Bioactive Compounds and HealthPromoting Properties of Berry Fruits: A Review. Plants Foods Hum Nutr. 63: 147-156.

Tähkäpää, S., Maijala, R., Korkeala, H., and Nevas, M. (2015). Patterns of food frauds and adulteration reported in the EU rapid alarm system for food and feed and in Finland. Food Control 47: 175-184.

Watson, R.R., and Preedy, V.R. (Ed.). (2010). Bioactive Foods in Promoting Health - Fruits and Vegetables. Academic Press Elsevier, London, United Kingdom, p. 656. ISBN: 978-0-12-374628-3. 